\title{
Graphene-templated directional growth of an inorganic nanowire
}

\section{Citation}

Lee, Won Chul, Kwanpyo Kim, Jungwon Park, Jahyun Koo, Hu Young Jeong, Hoonkyung Lee, David A. Weitz, Alex Zettl, and Shoji Takeuchi. 2015. “Graphene-Templated Directional Growth of an Inorganic Nanowire." Nature Nanotechnology 10 (5) (March 23): 423-428. doi:10.1038/ nnano.2015.36.

\section{Published Version}

doi:10.1038/nnano.2015.36

\section{Permanent link}

http://nrs.harvard.edu/urn-3:HUL.InstRepos:34310004

\section{Terms of Use}

This article was downloaded from Harvard University's DASH repository, and is made available under the terms and conditions applicable to Other Posted Material, as set forth at http:// nrs.harvard.edu/urn-3:HUL.InstRepos:dash.current.terms-of-use\#LAA

\section{Share Your Story}

The Harvard community has made this article openly available.

Please share how this access benefits you. Submit a story.

\section{Accessibility}




\section{Graphene-templated directional growth of}

\section{an inorganic nanowire}

4 Won Chul Lee ${ }^{1,2 \dagger *}$, Kwanpyo Kim ${ }^{3,4 \dagger}$, Jungwon Park ${ }^{5,6 \dagger}$, Jahyun $\mathrm{Koo}^{7}$, Hu Young Jeong ${ }^{8}$,

$5 \quad$ Hoonkyung Lee ${ }^{7}$, David A. Weitz ${ }^{5,6}$, Alex Zettl ${ }^{3,9 *}$, and Shoji Takeuchi ${ }^{1,2 *}$

${ }^{1}$ Institute of Industrial Science, The University of Tokyo, Tokyo 153-8505, Japan.

${ }^{2}$ ERATO Takeuchi Biohybrid Innovation Project, Japan Science and Technology Agency, Tokyo 153-

9 8904, Japan.

$10{ }^{3}$ Department of Physics, University of California at Berkeley, Berkeley, CA 94720, USA.

$11{ }^{4}$ Department of Physics, Ulsan National Institute of Science and Technology (UNIST), Ulsan 689-

12798 , South Korea.

$13{ }^{5}$ School of Engineering and Applied Sciences, Harvard University, Cambridge, MA 02138, USA.

$14{ }^{6}$ Department of Physics, Harvard University, Cambridge, MA 02138, USA.

$15 \quad{ }^{7}$ Department of Physics, Konkuk University, Seoul 143-701, South Korea.

${ }^{8}$ UNIST Central Research Facilities (UCRF), Ulsan National Institute of Science and Technology

(UNIST), Ulsan 689-798, South Korea

${ }^{9}$ Materials Sciences Division, Lawrence Berkeley National Laboratory, Berkeley, CA 94720, USA.

20 These authors equally contributed to this work.

$21 *$ *e-mails: takeuchi@iis.u-tokyo.ac.jp,wclee@iis.u-tokyo.ac.jp, and azettl@berkeley.edu. 
Assembling inorganic nanomaterials on graphene ${ }^{1-3}$ holds great promise in nanodevices and nanocomposite materials. Adding alignments in the assembly is expected to advance its functionalities ${ }^{4}$, as previously demonstrated in organic nanomaterials epitaxially aligned on graphitic surfaces $^{5-10}$. However, graphene's chemical inertness ${ }^{2,11-16}$ makes it challenging to assemble inorganic nanomaterials on pristine graphene, and even more challenging to align them orderly; previous techniques ${ }^{2,3}$ based on dangling bonds of damaged graphene ${ }^{11,17-21}$, intermediate seed materials ${ }^{11,15,16,22-24}$, and vapour-phase deposition at high temperature ${ }^{12-14,25-}$ ${ }^{28}$ only form randomly-oriented or poorly-aligned inorganic nanostructures on graphene derivatives. Here we report an inorganic nanowire that grows directly on pristine graphene, aligning itself with zigzag lattice directions of the graphene. This nanowire of gold(I) cyanide is synthesized by the self-organized growth in an aqueous solution at room temperature, which indicates that the inorganic material spontaneously binds to pristine graphene surfaces. Our first-principles calculations suggest that this unprecedented assembly originates from lattice matching and $\pi$-interaction to Au atoms between the two materials. Using the template of synthesized nanowires, we also fabricate nanostructures with controlled crystal orientations such as graphene nanoribbons with zigzag-edged directions.

The nanowires are synthesized by incubating single-layered graphene and solid gold simultaneously in an aqueous solution of $250 \mathrm{mM}$ ammonium persulfate, $\left(\mathrm{NH}_{4}\right)_{2} \mathrm{~S}_{2} \mathrm{O}_{8}$, at room temperature for 17 hours (Fig. 1a). Various types of gold precursors, such as gold nanoparticles or gold microstructures, can be used in this reaction depending on the experimental goal. The acidic solution of ammonium persulfate oxidizes gold precursors to form nanowires. Graphene, as both a substrate and template for the nanowire growth, is floated on the reaction solution, thus providing a surface on which the nucleation and growth of nanowires occur (Supplementary Methods and Supplementary Fig. S1). 
During this incubation, the nanowires grow on graphene surfaces along the specific lattice directions of graphene. Typical TEM (transmission electron microscopy) images of the graphene/nanowire samples (Fig. 1b and Supplementary Fig. S2a, b) show horizontally grown nanowires on their surfaces. The nanowires have the shape of nanoribbons laid down onto graphene surfaces, with a length, width, and thickness of $94.7 \pm 42.2 \mathrm{~nm}, 10.1 \pm 5.0 \mathrm{~nm}$, and $3.29 \pm 0.47 \mathrm{~nm}$, respectively, based on TEM and AFM measurements (Supplementary Fig. S3). Interestingly, the synthesized nanowires are oriented preferentially along three directions with rotations of $120^{\circ}$ relative to each other (Fig. 1b and Supplementary Fig. S2a-d). From the observed symmetry of nanowire axis directions, we expect that the nanowires have preferential growth directions related to the underlying graphene lattice structures. Indeed, the SAED (selected area electron diffraction) pattern (inset in Fig. 1b) of the samples clearly shows this epitaxial relationship between the nanowires and graphene. The nanowire axis directions in Fig. 1b show good orientational alignment to second-order diffraction peaks, (1-210) peaks, of graphene (circled in red). This alignment, which is also confirmed statistically (Fig. 1d), indicates that the nanowire directions coincide with the zigzag-edged directions of underlying graphene in real lattice space. In addition, diffraction peaks from nanowires (circled in yellow) are also aligned to graphene's (1-210) peaks (circled in red) in the SAED pattern. Together with high-resolution TEM images and their Fourier transforms (Fig. 1c and Supplementary Fig. S2e, f), the SAED pattern shows that each nanowire is single crystalline and that crystal lattices of the nanowires and graphene are rotationally aligned. An atomic resolution TEM image (Fig. 1e, f) directly confirms the nanowire alignment on graphene; the nanowire axis is aligned along the zigzag-edged direction of graphene lattice.

This nanowire alignment enables us to easily visualize crystal directions and grain boundaries in polycrystalline graphene using TEM or even SEM (scanning electron microscopy), as shown in Fig. 
$1 \mathrm{~g}, \mathrm{~h}$. Because nanowire axes directly represent the crystal directions of underlying graphene, tilt graphene grain boundaries can be identified by changes in the nanowire axis directions (Fig. 1i). Previously, atomic resolution imaging tools such as TEM and STM (scanning tunneling microscopy) have been utilized to directly image crystal directions and graphene grain boundaries ${ }^{29,30}$. However, these imaging processes often involve special sample preparation or substrate requirements and can be time-consuming. The present SEM-based imaging provides a facile tool to monitor crystal directions and graphene grain boundaries (spatial resolution of $\sim 100 \mathrm{~nm}$ from Supplementary Fig. S4), which is essential for studying the polycrystallinity of graphene and its implications in various properties.

We identify the nanowire material as gold(I) cyanide ${ }^{31}, \mathrm{AuCN}$, based on our elemental analysis and atomic structure characterizations. Elemental analyses including energy dispersive X-ray spectroscopy (EDX) and electron energy loss spectroscopy (EELS) in Supplementary Figs. S5 and $\mathrm{S} 6$ confirm the presence of $\mathrm{Au}$ and $\mathrm{N}$, along with non-graphene $\mathrm{C}$ (and a trace amount of $\mathrm{H}$ ) between all potential constituent elements from the precursor solution (Supplementary text). Atomicresolution TEM imaging (Fig. 2a and Supplementary Fig. S7) and SAED from multiple imaging axes (Fig. 2c, e and Supplementary Fig. S8) allow us to precisely determine the crystal structure. The atomic-resolution TEM images including Fig. 2a directly show unique atomic structures with two orthogonal lattice spacings of $5.08 \pm 0.01 \AA\left(\mathrm{d}_{1}\right)$ and $3.00 \pm 0.12 \AA\left(\mathrm{d}_{2}\right)$, which correspond to AuCN's hexagonal crystal (5.091 $\AA$ and $2.937 \AA$ ). Indeed, $\mathrm{AuCN}$ is the sole material candidate that satisfies the observed crystal symmetry and lattice spacings among reported inorganic compounds composed of possible constituent elements, Au, N, C, and/or H classified from the spectroscopic elemental analysis. We further validate that the post-simulated TEM image (Fig. 2c) of an AuCN crystal (Fig. 2b) reproduces the unique crystal patterns observed in the experimental TEM images. Most of all, SAED with multiple imaging axes confirms that the nanowire crystal is AuCN. Due to 
the nature of alignment between the nanowires and graphene, SAED patterns from the nanowires with no specimen tilting only display the specific d-spacing $(5.08 \AA)$ and its high-order peaks from a [100] zone axis (Fig. 2d and Supplementary Fig. S8a). Tilting multiple nanowires within the field of view allows us to investigate a set of SAED peaks from various zone axes and to precisely obtain three-dimensional structural information (Fig. 2e and Supplementary Fig. S8b). All of d-spacings measured from the SAED patterns of nanowires coincide well with those of AuCN (Supplementary Fig. S8c).

The crystal structure also reveals the alignment mechanism between the nanowires and graphene. Because it is well known that lattice matching between two materials causes heteroepitaxial alignment $^{13,14,27}$, we compare in-plane atomic configurations of the nanowires and graphene (Fig. 3a). Along nanowire axis directions, the unit cell size $\left(\mathrm{d}_{1}\right)$ of $\mathrm{AuCN}, 5.08 \pm 0.01 \AA$, coincides well with the length of two carbon hexagons, $4.92 \AA$, along graphene zigzag directions (lattice mismatch $=3.3$ $\pm 0.2 \%)$. In the nanowire width directions, the unit cell sizes $\left(6 \mathrm{~d}_{2}\right)$ of the nanowire material $(18.00 \pm$ $0.72 \AA$ ) and graphene $(19.17 \AA$ ) are also matched (lattice mismatch $=6.1 \pm 3.8 \%$ ). Note that the epitaxial alignment of inorganic materials on graphene is successful with a lattice mismatch of $\sim 2.9 \%\left(\mathrm{Bi}_{2} \mathrm{Se}_{3}\right.$ on graphene $\left.{ }^{13}\right)$ and is possible even with a lattice mismatch of $\sim 28 \%\left(\mathrm{MoS}_{2}\right.$ on graphene $^{27}$ ). Thus, in-plane lattice matching is mainly responsible for the epitaxial alignment between the AuCN nanowires and graphene.

We also confirm that the AuCN nanowires are likely to form directly on pristine surfaces of graphene, although other inorganic materials preferentially attach to dangling bonds such as graphene edges and defects ${ }^{2,11,13-17}$. First, Raman spectra of graphene before and after the nanowire synthesis (Supplementary Fig. S9a, b) maintain very low D peaks, which indicates that the graphene is of high quality and measurable defects are not introduced during the nanowire synthesis process. 
Second, atomic-resolution TEM imaging directly shows the pristine state of graphene underneath the nanowires. We strip off a nanowire from graphene (Supplementary Fig. S9c, d), and observe clean graphene lattice without any visible defects (Supplementary Fig. S9e). Third, we synthesize nanowires on sub-monolayer graphene samples consisting of domains disconnected from each other.

The sub-monolayer graphene transferred onto amorphous carbon films of TEM grids provides three different types of carbon surfaces for nanowire growth: pristine graphene in the middle of the domains, graphene defects at the edge of the domains, and amorphous carbon (from TEM grids) outside of the domains. During synthesis, no nanowire is formed on amorphous carbon surfaces and the nanowire density is uniform throughout the graphene domains and edges (Supplementary Fig. S9f-h). These results imply that the nanowires grow preferentially on pristine graphene surfaces (not on graphene defects) and do not perturb the crystal structure of graphene. Moreover, the third experiment (Supplementary Fig. S9f-h) indicates that graphene is a unique substrate promoting the formation of the uncommon inorganic crystals. As also shown by the synthesis of new organic crystals on graphene $e^{5,7,8}$, this result suggests the possibility of employing graphene as a template for advanced nanostructuring of inorganic materials.

The nanowire growth on pristine graphene suggests that the interaction between the two materials is unusual for inorganic materials. Due to the chemical inertness of graphene, the only way previously shown to synthesize inorganic structures directly on pristine graphene has been vapourphase deposition ${ }^{12-14,25-28}$, performed mostly at high temperature $\left(400{ }^{\circ} \mathrm{C}-900{ }^{\circ} \mathrm{C}\right)$. However, the reaction in this study occurs in aqueous phase at room temperature, which indicates that the interaction between the nanowires and pristine graphene provides a sufficient driving force for assembly without high energy. We further investigate the interaction between the nanowires and graphene using first-principles calculations (Supplementary Methods). In the optimized atomic configuration (Fig. 3a, b), both of AuCN's hexagonal crystal and graphene's sp ${ }^{2}$ carbon structures 
remain intact and their interlayer distance (3.29 $\AA)$ is almost the same as the interlayer distance (3.31

$2 \AA$ ) between $\mathrm{Au}(111)$ and graphene ${ }^{32}$. The parallel atomic structure at the interface (Fig. 3b, d)

3 implies that $\mathrm{Au}$ atoms, whose covalent radius $(1.3 \AA)$ is significantly larger than those $(0.7 \AA)$ of $\mathrm{C}$

4 and $\mathrm{N}$, mostly contribute to the interaction between the nanowire and graphene. The major binding

5 contribution of $\mathrm{Au}$ atoms is also confirmed in the charge density difference (Fig. 3d), where transferred electrons are localized only near $\mathrm{Au}$ atoms. However, the interaction between graphene and $\mathrm{Au}$ atoms in $\mathrm{AuCN}$ has unique characteristics unlike the common physical interaction between graphene and $\mathrm{Au}(111)$. The calculated binding energy of $\mathrm{AuCN}$ on graphene is $181 \mathrm{meV} / \mathrm{Au}$, significantly greater than $80 \mathrm{meV} / \mathrm{Au}$ for the binding energy between $\mathrm{Au}(111)$ and graphene via electrostatic interaction ${ }^{32}$. The charge density difference (Fig. 3d) shows that the $\pi$ orbitals of graphene donate electrons to $\mathrm{Au}$ atoms in $\mathrm{AuCN}$. These characteristics support that the nanowiregraphene interaction is mainly attributed to electron transfer between a transition metal (Au) and cyclic $\pi$-systems. This type of interaction, previously studied in organometallic chemistry, differs networks ${ }^{2,33}$. orientations (Fig. 4a). First, graphene nanoribbons with zigzag-edged directions, which can be important components for spintronic devices ${ }^{34}$, are fabricated by simply employing the synthesized 
1 plasma etching (Fig. 4b), and then the nanowires are removed with a $\mathrm{NaOH}$ solution without damaging the graphene (Fig. 4c and Supplementary Fig. S10). Because the nanowires are conformally attached on graphene and their widths can be controlled to within less than $10 \mathrm{~nm}$, the present method readily enables us to fabricate graphene nanoribbons with sub-10 nm widths. The AFM image (Fig. 4d) and its height profiles (Figs. 4e, f) indicate that the widths of the fabricated graphene nanoribbons are near $10 \mathrm{~nm}$ with the tip-size effect being considered. In addition, the thickness of the fabricated graphene nanoribbons is measured as $\sim 1 \mathrm{~nm}$, which is consistent with the reported thickness ${ }^{35}$ of single-layered graphene on $\mathrm{SiO}_{2}$. The Raman spectrum (Fig. 4g) also indicates that the graphene nanoribbons are successfully fabricated. The intensity ratio of the D and G bands $\left(I_{D} / I_{G}\right)$, a common measure to evaluate the quality of carbon materials, is measured as $\sim 0.67$, which is advanced for graphene nanoribbons produced by top-down fabrication. Overall, AFM and Raman data indicate that the fabricated graphene nanoribbons are of reasonable quality (particularly edge smoothness) compared to previously available graphene nanoribbons ${ }^{35}$. The crucial point in this fabrication process is that the directional alignment of the nanowires allows us to fabricate graphene nanoribbons selectively in zigzag-edged directions. Note that it has been difficult to fabricate graphene nanoribbons with zigzag-edged directions and only few previous studies ${ }^{36}$ have achieved it.

As the second example of crystallographically-aligned nanostructures, we fabricate gold nanoparticle chains that are aligned to the crystal directions of graphene substrates by decomposing the nanowires to gold nanoparticles (Fig. 4a). The electron beam in TEM is used to decompose the synthesized nanowires on graphene (Supplementary Video 1 and Fig. 4h). Gold nanoparticles (dark dots) grow near the nanowire and finally replace the entire nanowire (Supplementary Fig. S11). Applying heat $\left(200{ }^{\circ} \mathrm{C}, 30 \mathrm{~min}\right)$ can also decompose the nanowires to gold nanoparticles (Fig. $\left.4 \mathrm{i}, \mathrm{j}\right)$. Because the nanowire arrangement determines gold nanoparticle positions, the gold nanoparticle chains are naturally aligned along the zigzag lattice directions of graphene substrates (Fig. 4k, 1). 
1 These gold nanoparticle chains are in the optimal dimension for near-field plasmon coupling ${ }^{37}$,

2 which can propagate and/or enhance electromagnetic waves of applied light along the chain axes.

3 Thus, light with particular wavelength and polarization would interact strongly along specific crystal

4 directions of graphene. This phenomenon suggests potential applications in optical measurements of

5 graphene crystal directions and plasmonic sensing platforms.

6

7 In summary, we present the self-organized growth of inorganic AuCN nanowires that are readily 8 aligned to the zigzag lattice directions of single-layered pristine graphene. The direct alignment can

9 be utilized to extract and control crystallographic information of nanostructures, thus enabling us to 10 fabricate graphene nanoribbons with zigzag-edged directions. The synthetic method we introduce 11 demonstrates the possibility of employing graphene as a template for advanced classes of inorganic 12 nanomaterials even with wet chemistry. Furthermore, the unique interaction found in this study may 13 provide a new direction in fabricating graphene-inorganic heterostructures with intrinsic interface 14 properties. 


\section{References}

1 Geim, A. K. \& Grigorieva, I. V. Van der Waals heterostructures. Nature 499, 419-425 (2013).

2 Georgakilas, V. et al. Functionalization of graphene: covalent and non-covalent approaches, derivatives and applications. Chem. Rev. 112, 6156-6214 (2012).

3 Huang, X., Qi, X., Boey, F. \& Zhang, H. Graphene-based composites. Chem. Soc. Rev. 41, 666686 (2012).

4 Barth, J. V., Costantini, G. \& Kern, K. Engineering atomic and molecular nanostructures at surfaces. Nature 437, 671-679 (2005).

5 Hong, G. et al. Recent progress in organic molecule/graphene interfaces. Nano Today 8, 388-402 (2013).

6 Kim, D. W., Kim, Y. H., Jeong, H. S. \& Jung, H.-T. Direct visualization of large-area graphene domains and boundaries by optical birefringency. Nat. Nanotechnol. 7, 29-34 (2012).

7 Garnica, M. et al. Long-range magnetic order in a purely organic 2D layer adsorbed on epitaxial graphene. Nat. Phys. 9, 368-374, doi:Doi 10.1038/Nphys2610 (2013).

8 Colson, J. W. et al. Oriented 2D covalent organic framework thin films on single-layer graphene. Science 332, 228-231 (2011).

9 Zhang, F. et al. Epitaxial growth of peptide nanofilaments on inorganic surfaces: Effects of interfacial hydrophobicity/hydrophilicity. Angew. Chem. Int. Edit. 45, 3611-3613 (2006).

10 Fukushima, T. et al. Molecular ordering of organic molten salts triggered by single-walled carbon nanotubes. Science 300, 2072-2074 (2003).

11 Chung, K., Lee, C.-H. \& Yi, G.-C. Transferable GaN layers grown on ZnO-coated graphene layers for optoelectronic devices. Science 330, 655-657 (2010).

12 Addou, R., Dahal, A. \& Batzill, M. Growth of a two-dimensional dielectric monolayer on quasifreestanding graphene. Nat. Nanotechnol. 8, 41-45 (2013).

13 Dang, W., Peng, H., Li, H., Wang, P. \& Liu, Z. Epitaxial heterostructures of ultrathin topological insulator nanoplate and graphene. Nano Lett 10, 2870-2876 (2010).

14 Hong, Y. J. et al. Van der Waals Epitaxial Double Heterostructure: InAs/Single-Layer Graphene/InAs. Adv. Mater. 25, 6847-6853 (2013).

15 Wang, X., Tabakman, S. M. \& Dai, H. Atomic layer deposition of metal oxides on pristine and functionalized graphene. $J$ Am Chem Soc 130, 8152-8153 (2008).

16 Alaboson, J. M. P. et al. Templating sub-10 nm atomic layer deposited oxide nanostructures on graphene via one-dimensional organic self-assembled monolayers. Nano Lett 13, 5763-5770 (2013).

$17 \mathrm{Kim}$, K. et al. Selective metal deposition at graphene line defects by atomic layer deposition. Nat. Commun. 5, 4781 (2014).

18 Liang, Y. et al. Co3O4 nanocrystals on graphene as a synergistic catalyst for oxygen reduction reaction. Nat. Mater. 10, 780-786 (2011).

$19 \mathrm{Si}$, Y. \& Samulski, E. T. Exfoliated graphene separated by platinum nanoparticles. Chem. Mater. 20, 6792-6797 (2008).

20 Wang, H. et al. Mn3O4-graphene hybrid as a high-capacity anode material for lithium ion batteries. J Am Chem Soc 132, 13978-13980 (2010).

21 Lightcap, I. V., Kosel, T. H. \& Kamat, P. V. Anchoring Semiconductor and Metal Nanoparticles on a Two-Dimensional Catalyst Mat. Storing and Shuttling Electrons with Reduced Graphene Oxide. Nano Lett. 10, 577-583 (2010).

22 Huang, X. et al. Synthesis of hexagonal close-packed gold nanostructures. Nat. Commun. 2, 292 (2011).

23 Choi, D. et al. Fully rollable transparent nanogenerators based on graphene electrodes. $A d v$. Mater. 22, 2187-2192 (2010). 
24 Huang, X. et al. Reduced graphene oxide-templated photochemical synthesis and in situ assembly of Au nanodots to orderly patterned Au nanodot chains. Small 6, 513-516 (2010).

25 Rasche, B. et al. Stacked topological insulator built from bismuth-based graphene sheet analogues. Nat. Mater. 12, 422-425 (2013).

26 Kumar, B. et al. Controlled growth of semiconducting nanowire, nanowall, and hybrid nanostructures on graphene for piezoelectric nanogenerators. ACS nano 5, 4197-4204 (2011).

27 Shi, Y. et al. Van der Waals epitaxy of MoS2 layers using graphene as growth templates. Nano Lett 12, 2784-2791 (2012).

28 Zhou, H. et al. The transformation of a gold film on few-layer graphene to produce either hexagonal or triangular nanoparticles during annealing. Carbon 52, 379-387 (2013).

29 Huang, P. Y. et al. Grains and grain boundaries in single-layer graphene atomic patchwork quilts. Nature 469, 389-392 (2011).

$30 \mathrm{Kim}, \mathrm{K}$. et al. Grain boundary mapping in polycrystalline graphene. ACS nano 5, 2142-2146 (2011).

31 Hibble, S. J., Hannon, A. C. \& Cheyne, S. M. Structure of AuCN determined from total neutron diffraction. Inorg. Chem. 42, 4724-4730 (2003).

32 Giovannetti, G. et al. Doping graphene with metal contacts. Phys Rev Lett 101, 026803 (2008).

33 Sarkar, S. et al. Organometallic hexahapto functionalization of single layer graphene as a route to high mobility graphene devices. Adv. Mater. 25, 1131-1136 (2013).

34 Son, Y.-W., Cohen, M. L. \& Louie, S. G. Half-metallic graphene nanoribbons. Nature 444, 347349 (2006).

35 Jiao, L., Zhang, L., Wang, X., Diankov, G. \& Dai, H. Narrow graphene nanoribbons from carbon nanotubes. Nature 458, 877-880 (2009).

36 Shi, Z. et al. Patterning graphene with zigzag edges by self-aligned anisotropic etching. $A d v$. Mater. 23, 3061-3065 (2011).

37 Halas, N. J., Lal, S., Chang, W. S., Link, S. \& Nordlander, P. Plasmons in strongly coupled metallic nanostructures. Chem. Rev. 111, 3913-3961 (2011). 
Acknowledgements We thank A.P. Alivisatos, H. Fujita, Y. Arakawa, B.J. Kim, L. Yang, J. Moon, Y. Ota, H. Suh, J. Kwon, and J. Min for helpful discussions. We also thank J. Kim and Y. Mizutani for the AFM analysis, S. Mori and M. Onuki for technical supports, and A. Sato for help with graphic illustrations. This work was mainly supported by the Takeuchi Biohybrid Innovation Project, Exploratory Research for Advanced Technology (ERATO), Japan Science and Technology (JST). A.Z. and K.K. gratefully acknowledge support from the Director, Office of Energy Research, Materials Sciences and Engineering Division, of the US Department of Energy (DE-AC0205CH11231) and from the Office of Naval Research (MURI grant N00014-09-1066). K.K. also acknowledges support from the 2014 Research Fund (1.140060.01) of UNIST. D.A.W and J.P gratefully acknowledge support from the Harvard MRSEC (DMR-0820484) and Amore-Pacific. H.L. and J.K. gratefully acknowledge support from the Basic Science Research Program (KRF2012R1A1A1013124) of the National Research Foundation of Korea under the Ministry of Education, Science and Technology.

Author contributions W.C.L, K.K., and J.P. conceived the design of the study. S.T., A.Z., and D.A.W. supervised the project. K.K. and J.P initially discovered the nanowire synthesis phenomenon. W.C.L, K.K., J.P., and H.Y.J. performed all experiments. J.K. and H.L. performed first-principles calculations. W.C.L, K.K., J.P., H.L., D.A.W., A.Z., and S.T. wrote the manuscript. All authors discussed the results and commented on the manuscript.

Author Information Supplementary information is available in the online version of the paper. Reprints and permissions information is available at www.nature.com/reprints. Correspondence and requests for materials should be addressed to S.T., W.C.L., or A.Z.

Competing financial interests The authors declare no competing financial interests. 

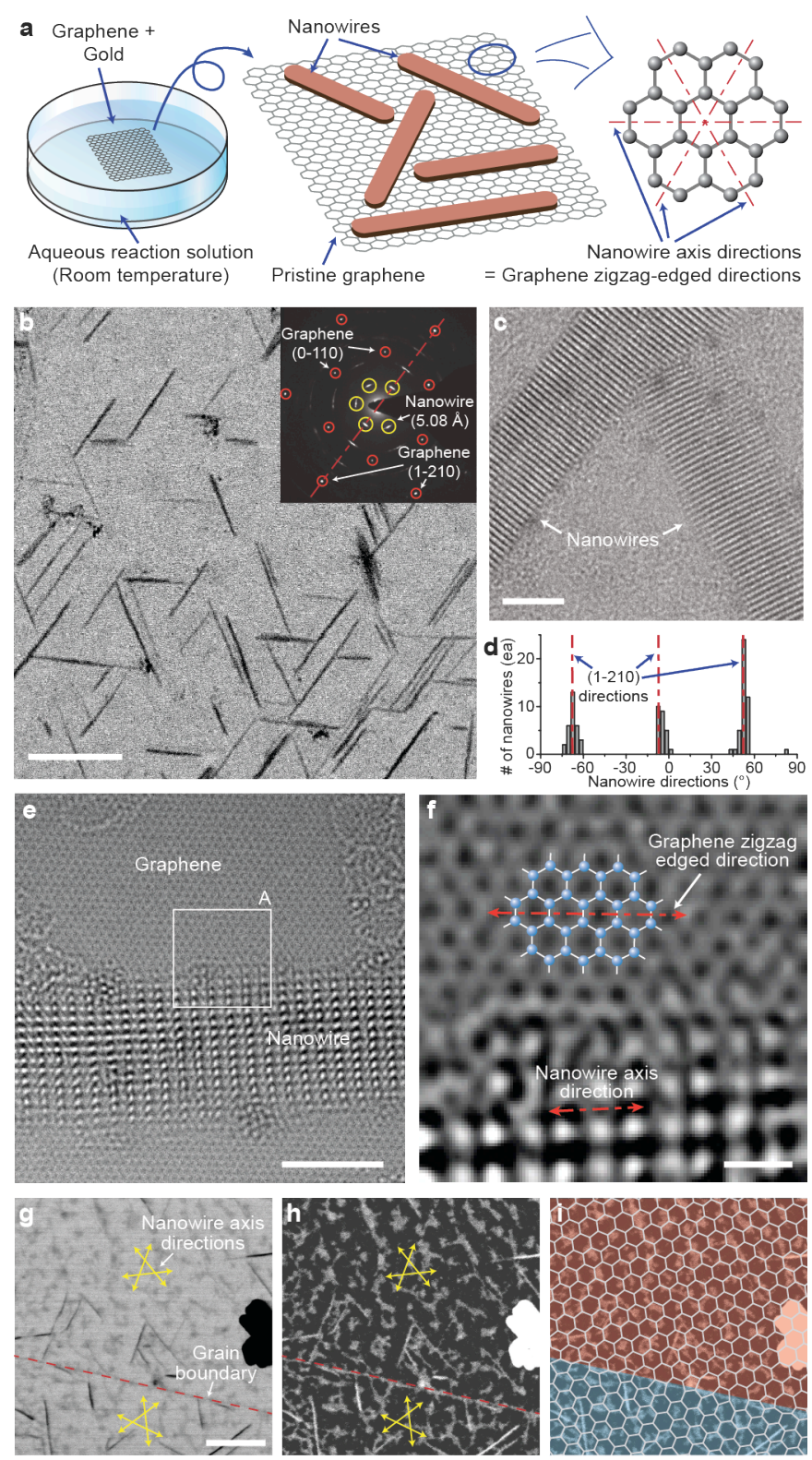

Figure 1. Directional growth of inorganic nanowires on graphene. a, Schematic illustration of the process. An aqueous-phase reaction at room temperature synthesizes nanowires whose axes are parallel to the zigzag lattice directions of pristine graphene. b, TEM image of the synthesized nanowires on graphene. Scale bar: $100 \mathrm{~nm}$. The inset shows an SAED pattern of the nanowiregraphene sample. The nanowire axes are aligned to the zigzag lattice directions, (1-210) directions, of graphene. c, High-resolution TEM image of the nanowires. Scale bar: $5 \mathrm{~nm}$. d, Histogram of the angular distributions of the nanowire axes in $\mathbf{b}$. e, Atomic-resolution TEM image of a nanowire on graphene. Scale bar: $3 \mathrm{~nm}$. f, Enlarged view of the section A in e. Low-pass filtering (cut-off: 4 pixels) is applied to remove high-frequency noise. The directional alignment of the nanowire axis to the graphene zigzag lattice direction is clearly visualized. Scale bar: $0.5 \mathrm{~nm}$. $\mathbf{g}-\mathbf{i}$, Nanowire-based imaging of crystal directions and domain boundaries of polycrystalline graphene. The same specimen area is imaged with TEM (g) and SEM (h). The graphene lattice directions can be identified using the nanowire axis directions. Scale bar: $100 \mathrm{~nm}$. i, SEM image (h) with an overlay of the pseudo-lattice structures of graphene. The red and blue colour maps represent different domains with relatively tilted lattice directions. 


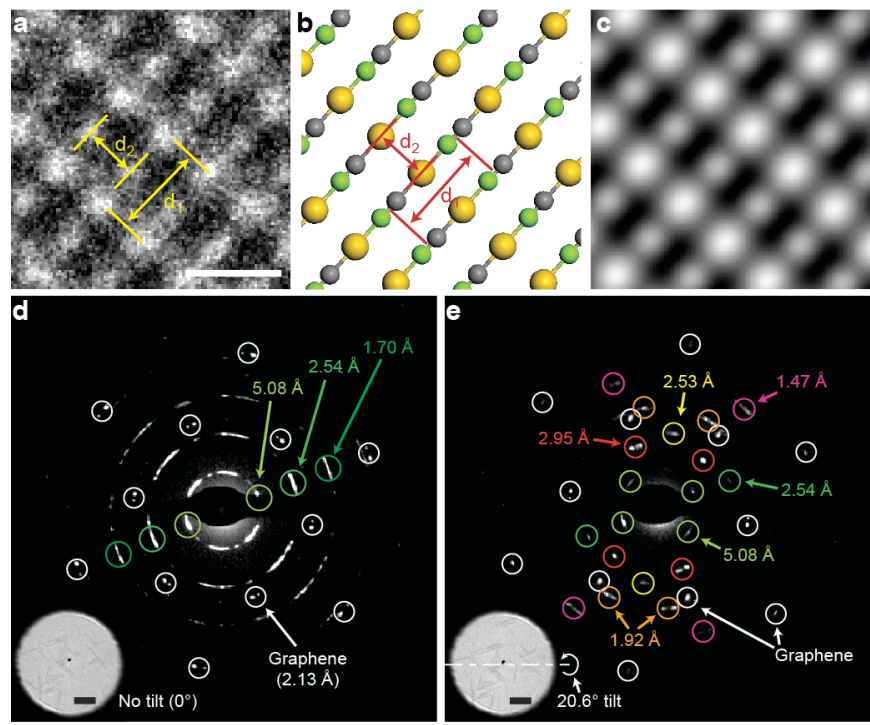

Figure 2. Atomic-resolution TEM imaging and SAED of the nanowires. a, Atomic-resolution TEM image of the nanowire. The two lattice spacings along $\left(\mathrm{d}_{1}\right)$ and perpendicular $\left(\mathrm{d}_{2}\right)$ to the nanowire axis direction are measured as $5.08 \pm 0.01 \AA$ and $3.00 \pm 0.12 \AA$, respectively. The TEM image of larger area is shown in Supplementary Fig. S6. Scale bar: $0.5 \mathrm{~nm}$. b, Crystal structure of $\mathrm{AuCN}$. The yellow, green, and gray spheres represent gold, nitrogen, and carbon atoms, respectively. The lattice spacings $d_{1}$ and $d_{2}$ obtained from the crystal structure of AuCN are 5.091 $\AA$ and $2.937 \AA$, respectively. c, Simulated TEM image from the crystal structure in $\mathbf{b}$. The captured TEM image (a), crystal structure from first-principles calculations (b), and simulated TEM image (c) show good agreement. d-e, SAED patterns of a nanowire-graphene sample under no tilt (d) and a $20.6^{\circ}$ tilt (e). The insets show the sample areas where the SAED patterns are measured. Scale bar: $100 \mathrm{~nm}$. 
Figure 3. Interaction between the nanowire and graphene estimated by first-principles calculations. a-b, Optimized atomic configuration of the nanowire and graphene estimated by firstprinciples calculations. A plan-view from the top (a) and a side-view from the nanowire-axis direction (b) are displayed. $\mathbf{c}$, Schematic view of the nanowire and graphene. Red arrows represent the viewing orientations of Figs. a, $\mathbf{b}$, and d. d, Molecular structures with an overlay of CCD (charge density difference) isosurfaces at the nanowire-graphene interface. The red and blue CCD isosurfaces are contoured at the level of $\pm 0.0004 \mathrm{e} \AA^{-3}$, indicating electron accumulation and depletion, respectively. 
a

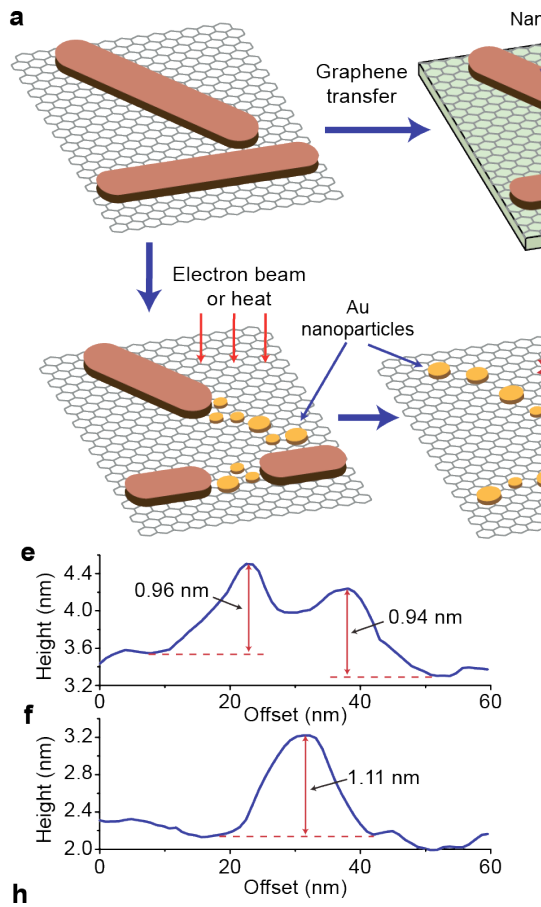

h

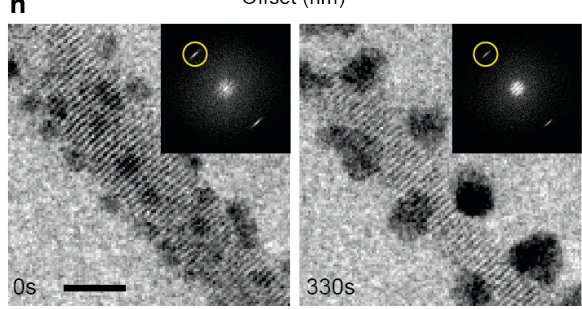

anowires
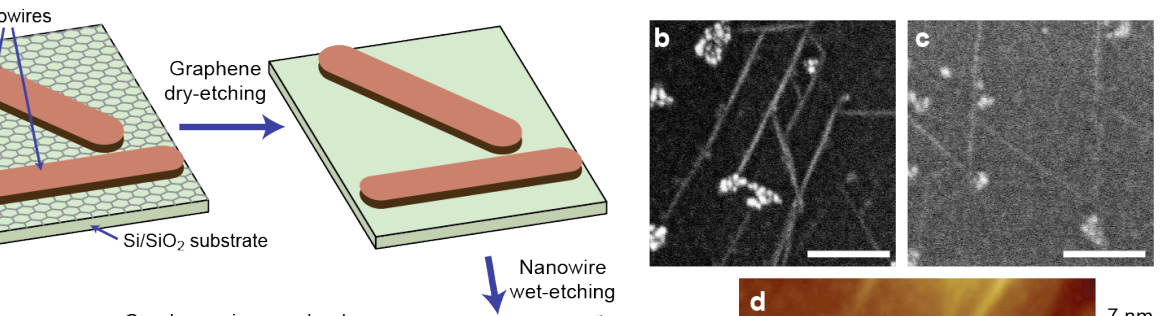

d
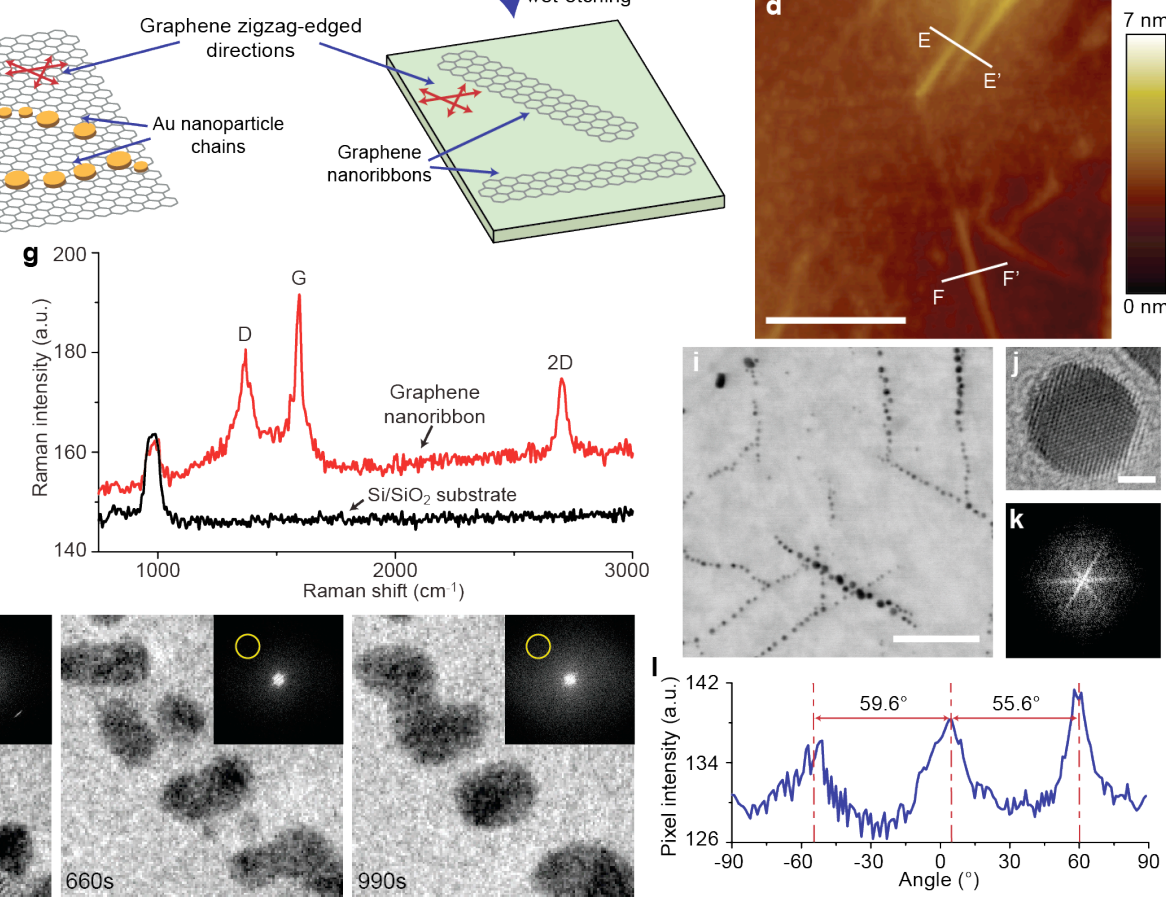

Figure 4. Fabrication of crystallographically-aligned nanostructures. a, Fabrication process for the graphene nanoribbons and Au nanoparticle chains based on the nanowires aligned on graphene. b-c, SEM images of the nanowires on graphene (b) and the fabricated graphene nanoribbons (c), which are aligned with the zigzag lattice directions. Scale bars: $100 \mathrm{~nm}$. d, AFM image of the graphene nanoribbons. Scale bar: $100 \mathrm{~nm}$. e-f, Height profiles of the sample along E-E' (e) and F-F' (f) lines in $\mathbf{d}$. g, Raman spectrum of the fabricated graphene nanoribbons (red line). The negativecontrol signal (black line) is measured from the position where graphene is fully etched out within the same sample. $\mathbf{h}$, A series of TEM images from Supplementary Video 1 showing the nanowire decomposition process to Au nanoparticle chains under e-beam irradiation. The insets show Fourier transforms of the TEM images. Scale bar: $5 \mathrm{~nm}$. i, Au nanoparticle chains formed by the thermal decomposition of nanowires. Scale bar: $100 \mathrm{~nm}$. j, High-resolution TEM image of an Au nanoparticle in the chain. Scale bar: $2 \mathrm{~nm}$. $\mathbf{k}$, Fourier transform of $\mathbf{i}$. The directions of the bright lines are orthogonal to the axis directions of the Au nanoparticle chains. $\mathbf{l}$, Radial pixel intensities of $\mathbf{k}$ averaged along a line from the centre at different angles. The directional alignment of the $\mathrm{Au}$ nanoparticle chains is shown with inter-peak angles of approximately $60^{\circ}$. 